\title{
Nonlocal dynamics and infinite non-relativistic conformal symmetries
}

\author{
K. Andrzejewski* \\ Department of Computer Science, \\ University of Łódź, \\ Pomorska 149/153, 90-236 Łódź, Poland \\ K. Bolonek-Lasoń ${ }^{\dagger}$ \\ Department of Statistical Methods, \\ University of Łódź, \\ Rewolucji 1905 St. 41/43, 90-214, Łódź, Poland
}

\begin{abstract}
We study the symmetry of the class of nonlocal models which includes the nonlocal extension of the Pais-Uhlenbeck oscillator. As a consequence, we obtain an infinite dimensional symmetry algebra, containing the Virasoro algebra, which can be considered as a generalization of the non-relativistic conformal symmetries to the infinite order. Moreover, this nonlocal extension resembles to some extent the string model and on the quantum level it leads to the centrally extended Virasoro algebra.
\end{abstract}

\footnotetext{
*E-mail: k-andrzejewski@uni.lodz.pl

†E-mail: kbolonek1@wp.pl
} 


\section{Introduction}

Because of the importance of the noncommutative field theories and the string field theory the nonlocal models have been extensively investigated in last two decades (see, e.g., [1]-[7], as a very brief list of references). However, the nonlocality in physics has a long history (see [8] and the references in [9]). The nonlocal systems provide an extension of the higher derivatives ones to the case of the infinite order derivatives. This fact is reflected in form of the Hamiltonian formalism for the nonlocal theories which is a generalization of the Ostrogradski approach to the higher derivatives theory [9]-[13].

The basic example of the theory with higher derivatives is the PaisUhlenbeck (PU) oscillator of order $N$ (which generalizes the standard Lagrangian of the harmonic oscillator to the case of the $N$-th order time derivative) proposed in the classical paper [8]. This model has attracted interest thought the years (for the last few years see, e.g., [14]-30]). In particular, it has been shown (see [30]) that when the relevant eigenfrequencies are proportional to consecutive odd integers then the maximal symmetry group of the PU oscillator is the $l$-conformal Newton-Hooke group with half-integer

$l=N-\frac{1}{2}$, which is isomorphic to the $l$-conformal Galilei group (for this reason we will simply refer to it as the l-conformal non-relativistic group in what follows). These groups are natural conformal extensions of the NewtonHooke (Galilei) group, (in particular for $N=1$ we obtain the Schrödinger one) and originally have been introduced in the context of non-relativistic gravity [31, 32]; however, there are many dynamical models, both classical (including also the second order dynamical equations) and quantum, which exhibit such symmetries [33]-41]. Furthermore, in a number of papers [42][49] infinite dimensional extensions of those symmetries, for fixed order $l$, have been also considered in the context of some more sophisticated models. Let us note that neither the $l$-conformal non-relativistic algebra nor its infinite extension are well defined as $N$ (or $l$ ) tends to infinity.

In the present paper we show that a very simple nonlocal theory (proposed in ref. [8]) enjoys symmetry which can be considered as an infinitedimensional generalization of the non-relativistic conformal symmetries to the case of $N$ going out to infinity. The starting point is the mentioned observation that the $\left(N-\frac{1}{2}\right)$-conformal non-relativistic group appears as a symmetry of the PU oscillator of order $N$. Next, we note that in ref. [8] the authors introduce (however, slightly formally) the $N=\infty$ extension of the PU oscillator which, in the case of odd frequencies, leads after some manip- 
ulations to the quadratic nonlocal Lagrangian (see also [13]). We find the symmetry algebra of this nonlocal Lagrangian and its central extension on the Hamiltonian level. The symmetry algebra obtained in this way contains two representations of the Virasoro algebra. In the case of many spatial dimensions we obtain the Virasoro-Kac-Mody type algebra. Finally, we show that on the quantum level the Virasoro generators do form the centrally extended algebra.

The paper is organized as follows. In Section 2 we describe in some detail a class of nonlocal Lagrangians and derive the explicit relation, on the Lagrangian level, between the symmetry and the integral of motion. In Section 3 we briefly recall the Hamiltonian formalism for the nonlocal PU model; in the next section, we find the symmetries and corresponding integrals of motion for this model in the Lagrangian framework. Section 5 is devoted to the symmetry realizations on the Hamiltonian and quantum levels with special emphasis put on the Virasoro algebra. Finally, Section 6 contains the final discussion and sketches the possible ways of further development.

\section{Nonlocal systems}

Let us consider the following class of nonlocal Lagrangians

$$
L=L(q(t), q(t+\alpha)), \quad \alpha>0 .
$$

The action principle

$$
\delta S \equiv \int_{-\infty}^{\infty} \frac{\delta L(s)}{\delta q(t)} d s=0,
$$

applied to the Lagrangian (2.1) implies the following equation of motion

$$
\frac{\partial L(q(t), q(t+\alpha))}{\partial q(t)}+\frac{\partial L(q(t-\alpha), q(t))}{\partial q(t)}=0 .
$$

In order to better understand the structure of the nonlocal theory and to derive the Hamiltonian formalism (and, eventually, the quantum theory) let us expand the Lagrangian (2.1) in powers of the parameter $\alpha$; then $L$ becomes a function of all time derivatives of $q(t)$. Thus it requires an infinite number of initial conditions or the knowledge of a finite piece of the trajectory. Consequently, we need a Hamiltonian formalism which would be a generalization of the Ostrogradski one which is valid only for a finite order derivatives. Such 
formalism has been proposed and discussed in refs. [9]-[13]. In this approach we consider, instead of the trajectory $q(t), 1+1$ dimensional field $Q(t, \lambda)$ such that

$$
Q(t, \lambda)=q(t+\lambda)
$$

Our Lagrangian $L$ is rewritten as follows

$$
\tilde{L}(Q(t, 0), Q(t, \alpha))=\int_{-\infty}^{\infty} d \lambda \delta(\lambda) \tilde{L}(Q(t, \lambda), Q(t, \lambda+\alpha)),
$$

where $\tilde{L}(Q(t, \lambda), Q(t, \lambda+\alpha))$ is obtained from the original Lagrangian (2.1) by making the following replacements

$$
q(t) \rightarrow Q(t, \lambda), \quad q(t+\alpha) \rightarrow Q(t, \lambda+\alpha) .
$$

Now, the Hamiltonian for the field $Q$ is provided by

$$
H=\int_{-\infty}^{\infty} d \lambda P(t, \lambda) Q^{\prime}(t, \lambda)-\tilde{L}
$$

where $P$ is the canonical momentum of $Q$ and prime stands for $\partial_{\lambda}$. The Poisson bracket is of the form

$$
\left\{Q(t, \lambda), P\left(t, \lambda^{\prime}\right)\right\}=\delta\left(\lambda-\lambda^{\prime}\right)
$$

In order to recover the original dynamics one has to impose new constraint:

$$
\phi \equiv P(t, \lambda)-\frac{1}{2} \int_{-\infty}^{\infty} d \sigma(\operatorname{sgn}(\lambda)-\operatorname{sign}(\sigma)) E(t, \sigma, \lambda) \approx 0
$$

where

$$
E(t, \sigma, \lambda)=\frac{\delta L(Q(t, \sigma), Q(t, \sigma+\alpha))}{\delta Q(t, \lambda)} .
$$

The constraint $\phi$ implies the secondary constrains which can be written collectively in the following form

$$
\psi \equiv \int_{-\infty}^{\infty} d \sigma E(t, \sigma, \lambda) \approx 0
$$

The constraint (2.11) is equivalent to the Euler-Lagrange equation for the nonlocal Lagrangian (2.1) and together with the constraint (2.9) they form 
the second class constraints. So we can use the Dirac method for constrained systems and solve $\phi$ for $P$. Then the Hamiltonian

$$
\begin{aligned}
H= & -\frac{1}{2} \int_{-\infty}^{\infty} d \lambda(\operatorname{sgn}(\lambda-\alpha)-\operatorname{sign}(\lambda)) \frac{\partial L(Q(t, \lambda-\alpha), Q(t, \lambda))}{\partial Q(t, \lambda)} Q^{\prime}(t, \lambda) \\
& -L(Q(t, 0), Q(t, \alpha)),
\end{aligned}
$$

is expressed only in terms of $Q$ which is subjected to the single condition (2.11) and satisfies commutation relations following from the relevant Dirac bracket.

Apart from the Hamiltonian we can look for another integrals of motion (and the corresponding symmetries) related to the Lagrangian (2.1). In order to do this let us consider the infinitesimal transformation of the time $t$ and the coordinate $q$

$$
t^{\prime}=t+\delta t(t), \quad q^{\prime}\left(t^{\prime}\right)=q(t)+\delta q(t),
$$

satisfying the following condition

$$
\delta t(t+\alpha)=\delta t(t)
$$

Then

$$
t^{\prime}+\alpha=t+\alpha+\delta t(t+\alpha), \quad q^{\prime}\left(t^{\prime}+\alpha\right)=q(t+\alpha)+\delta q(t+\alpha) .
$$

Next, let us define

$$
\delta_{0} q(t)=\delta q(t)-\dot{q}(t) \delta(t), \quad \delta_{0} q(t+\alpha)=\delta q(t+\alpha)-\dot{q}(t+\alpha) \delta t(t+\alpha) .
$$

The action principle is invariant under the transformations (2.13) provided

$$
L\left(q^{\prime}\left(t^{\prime}\right), q^{\prime}\left(t^{\prime}+\alpha\right)\right) \frac{d t^{\prime}}{d t}=L(q(t), q(t+\alpha))+\frac{d F}{d t},
$$

which infinitesimally takes the form

$$
\frac{d}{d t}(L \delta t-\delta F)+\frac{\partial L(q(t), q(t+\alpha))}{\partial q(t)} \delta_{0} q(t)+\frac{\partial L(q(t), q(t+\alpha))}{\partial q(t+\alpha)} \delta_{0} q(t+\alpha)=0 .
$$

Using eq. (2.18) one can check, by direct calculations, that the function $C$ defined as follows

$$
C=L \delta t+\int_{t}^{t+\alpha} d \lambda \frac{\partial L(q(\lambda-\alpha), q(\lambda))}{\partial q(\lambda)} \delta_{0} q(\lambda)-\delta F,
$$


is an integral of motion associated with the symmetry (2.13). This result can be also obtained following ref. [11] directly within the Hamiltonian framework keeping in mind that the constraint (2.9), being of second kind, can be used to eliminate the momentum variable $P(t, \lambda)$. Let us note that in the case of the time translation, i.e.,

$$
\delta t=-\epsilon, \quad \delta q=0
$$

$C$ coincides with $H$ after making the replacement (2.6)

\section{Nonlocal Pais-Uhlenbeck model}

The PU oscillator of order $N$

$$
L_{P U}=-\frac{1}{2} q \prod_{k=1}^{N}\left(\frac{d^{2}}{d t^{2}}+\omega_{k}^{2}\right) q,
$$

studied in the original paper [8] is considered there as a starting point towards the nonlocal theory. Namely, it has been shown (rather formally) that taking the limit $N \rightarrow \infty$ in (3.1) we obtain, in the case of the odd frequencies

$\omega_{k}=(2 k-1) \frac{\pi}{2 \alpha}, k=1,2, \ldots$, a quadratic nonlocal Lagrangian which (after some manipulations) can be written in the form

$$
L=-\frac{m}{\alpha^{2}} q(t) q(t+\alpha)
$$

where $m$ and $\alpha$ are some constants of the dimension of mass and time, respectively. Equation of motion (2.3) in this case yields

$$
q(t-\alpha)+q(t+\alpha)=0
$$

The slightly surprising interpretation of the Lagrangian (3.2) as a generalization of the PU oscillator with the odd frequencies to the case of the infinite time derivatives (or, equivalently, with infinite number of oscillators) has been recently confirmed, in two ways, in ref. [13]. First, it was shown that, expanding the right-hand side of (3.2) in a Taylor series in $\alpha$ and then restricting ourselves to the first $k$ terms we obtain a Lagrangian, which is proportional to the PU oscillator with some frequencies which tends to the odd ones in the limit $k \rightarrow \infty$. The authors of ref. [13] applied also the 
Hamiltonian formalism discussed in the previous section and arrive at the following Hamiltonian dynamics

$$
H=\frac{m}{2 \alpha^{2}} \int_{-\infty}^{\infty} d \lambda(\operatorname{sgn}(\lambda-\alpha)-\operatorname{sign}(\lambda)) Q(t, \lambda-\alpha) Q^{\prime}(t, \lambda)+\frac{m}{\alpha^{2}} Q(t, 0) Q(t, \alpha),
$$

where $Q(t, \lambda)$ is subjected to the condition

$$
\psi \equiv Q(t, \lambda-\alpha)+Q(t, \lambda+\alpha) \approx 0,
$$

and the Dirac bracket reads

$$
\left\{Q(t, \lambda), Q\left(t, \lambda^{\prime}\right)\right\}=\frac{\alpha^{2}}{m} \sum_{k=-\infty}^{\infty}(-1)^{k} \delta\left(\lambda-\lambda^{\prime}+(2 k+1) \alpha\right) .
$$

The constraint $\psi$ can be explicitly solved:

$$
Q(t, \lambda)=\sum_{k=-\infty}^{\infty} a_{k}(t) \psi_{k}(\lambda)
$$

where

$$
\psi_{k}=\frac{1}{\sqrt{2 \alpha}} e^{\frac{i \pi}{2 \alpha}(2 k+1) \lambda},
$$

form an orthonormal and complete set in $L^{2}[-\alpha, \alpha]$. Then the Hamiltonian (3.4), expressed in terms of the new variables, takes the form

$$
H=\frac{\pi m}{4 \alpha^{3}} \sum_{k=-\infty}^{\infty}(-1)^{k}(2 k+1) a_{k} a_{-(k+1)}
$$

while the Dirac bracket reads

$$
\left\{a_{k}, a_{n}\right\}=\frac{i \alpha^{2}}{m}(-1)^{k} \delta_{k+n+1,0} .
$$

After a simple redefinition of $a$ 's

$$
a_{k}=\frac{\alpha}{\sqrt{m}} \begin{cases}c_{k} & \text { k-odd positive } \\ c_{-(k+1)} & \text { k-odd negative } \\ \bar{c}_{k} & \text { k-even nonnegative } \\ \bar{c}_{-(k+1)} & \text { k-even negative }\end{cases}
$$


we arrive at the infinite alternating sum of oscillators with the odd frequencies

$$
H=\sum_{k=0}^{\infty}(-1)^{k}(2 k+1) \frac{\pi}{2 \alpha} \bar{c}_{k} c_{k},
$$

with the standard brackets

$$
\left\{c_{k}, \bar{c}_{n}\right\}=-i \delta_{k n}
$$

Thus the model (3.2) describes the PU oscillator of infinite order with the odd frequencies. This fact is interesting from the symmetry point of view because, as it was shown in [30], for the odd frequencies the symmetry group of the $N$-th order PU oscillator is larger than for the case of generic frequencies; it contains the conformal and dilatation generators which together with the remaining symmetry generators (related to the time translation and the change of initial conditions) form the $\left(N-\frac{1}{2}\right)$-conformal non-relativistic algebra. Consequently, the question arises about the symmetry of the nonlocal model (3.2).

\section{Symmetries of the nonlocal PU model on the Lagrangian level}

We start with the observation that the change of the initial conditions produce new solutions. Due to the fact that any solution of the equation of motion (3.3) is antiperiodic functions on the interval $[-\alpha, \alpha]$ we postulate the infinitesimal symmetry

$$
t^{\prime}=t, \quad q^{\prime}=q+\delta q, \quad \text { where } \delta q(t-\alpha)+\delta q(t+\alpha)=0 .
$$

Indeed, taking

$$
\delta F=-\frac{m}{\alpha^{2}} \int_{t}^{t+\alpha} d \lambda \delta q(\lambda-\alpha) q(\lambda),
$$

one can check that the symmetry condition (2.18) holds. The corresponding integral of motion reads

$$
C=-\frac{m}{\alpha^{2}} \int_{-\alpha}^{\alpha} q(\lambda+t-\alpha) \delta q(\lambda+t) .
$$


Expanding $\delta q$ in the Fourier serie (after substitution $q(t) \rightarrow q(t) e^{-\frac{i \pi t}{2 \alpha}}$ ) we can identify the symmetry generators corresponding to the coefficients in the Fourier expansion

$$
\delta q_{k}^{c}=\epsilon \cos \left(\frac{2 k+1}{2} \omega t\right), \quad \delta q_{k}^{s}=\epsilon \sin \left(\frac{2 k+1}{2} \omega t\right),
$$

where $\omega=\frac{\pi}{\alpha}$ and $k=0,1,2 \ldots$ (for simplicity of the further notation we can extend $k$ to the negative integers). Consequently, the symmetry generators take the form

$$
\begin{aligned}
& C_{k}^{c}=-\frac{m}{\alpha^{2}} \int_{-\alpha}^{\alpha} q(\lambda+t-\alpha) \cos \left(\frac{2 k+1}{2} \omega(\lambda+t)\right), \\
& C_{k}^{s}=-\frac{m}{\alpha^{2}} \int_{-\alpha}^{\alpha} q(\lambda+t-\alpha) \sin \left(\frac{2 k+1}{2} \omega(\lambda+t)\right),
\end{aligned}
$$

or in the differential realization as commuting operators

$$
C_{k}^{c}=-\cos \left(\frac{2 k+1}{2} \omega t\right) \frac{d}{d q}, \quad C_{k}^{s}=-\sin \left(\frac{2 k+1}{2} \omega t\right) \frac{d}{d q} .
$$

Note that with our convention concerning $k$ (positive and negative) some generators are linearly dependent.

Now, the main observation is that except the quite natural symmetry (4.1) the Lagrangian (3.2) possesses a more interesting set of symmetries related to the time transformation. Namely, let us consider an arbitrary time transformation $t^{\prime}=t^{\prime}(t)$ satisfying the condition

$$
t^{\prime}(t+\alpha)=t^{\prime}(t)+\alpha
$$

and define the following transformation of the coordinate $q$

$$
q^{\prime}\left(t^{\prime}\right)=q(t)\left(\frac{d t^{\prime}}{d t}\right)^{-\frac{1}{2}}
$$

Then one can check that the invariance condition (2.17) for the nonlocal Lagrangian (3.2) is satisfied (with $F=0$ ). In order to identify this symmetry let us take its infinitesimal form

$$
t^{\prime}=t+\epsilon f(t), \quad f(t+\alpha)=f(t) .
$$


Then

$$
\delta t=\epsilon f(t), \quad \delta_{0} q=-\frac{\epsilon}{2} \dot{f} q
$$

due to eq. (2.19) the corresponding integral of motion reads

$$
\begin{gathered}
C_{f}=-\frac{m}{\alpha^{2}} q(t) q(t+\alpha) f(t)+\frac{m}{\alpha^{2}} \int_{0}^{\alpha} d \lambda q(\lambda+t-\alpha) \\
\left(\frac{1}{2} f^{\prime}(\lambda+t) q(\lambda+t)+q^{\prime}(\lambda+t) f(\lambda+t)\right)= \\
\frac{m}{2 \alpha^{2}} \int_{-\alpha}^{\alpha} q(\lambda+t-\alpha) q^{\prime}(\lambda+t) f(\lambda+t),
\end{gathered}
$$

where in the second equality we have used (3.3). Since the function $f$ is periodic with the period $\alpha$ it can be expanded in terms of the functions

$$
f_{k}^{c}(t)=\frac{1}{2 \omega} \cos (2 k \omega t), \quad f_{k}^{s}(t)=\frac{1}{2 \omega} \sin (2 k \omega t)
$$

for $k=0,1,2 \ldots$ Substituting (4.12) into (4.11) we obtain a family of generators $L_{k}^{c}, L_{k}^{s}$ (as before we may assume $k$ integer, then $L_{-k}^{s}=-L_{k}^{s}$ and $\left.L_{-k}^{c}=L_{k}^{c}\right)$. One can easily find the differential realizations of those generators:

$$
\begin{aligned}
L_{k}^{c} & =\frac{-1}{2 \omega} \cos (2 k \omega t) \frac{d}{d t}-\frac{k}{2} \sin (2 k \omega t) q \frac{d}{d q} \\
L_{k}^{s} & =\frac{-1}{2 \omega} \sin (2 k \omega t) \frac{d}{d t}+\frac{k}{2} \cos (2 k \omega t) q \frac{d}{d q}
\end{aligned}
$$

as well as the commutation rules

$$
\begin{aligned}
& {\left[L_{k}^{s}, L_{n}^{s}\right]=\frac{k-n}{2} L_{n+k}^{s}+\frac{n+k}{2} L_{n-k}^{s}} \\
& {\left[L_{k}^{c}, L_{n}^{c}\right]=\frac{n-k}{2} L_{n+k}^{s}+\frac{n+k}{2} L_{n-k}^{s}} \\
& {\left[L_{k}^{s}, L_{n}^{c}\right]=\frac{k-n}{2} L_{n+k}^{c}+\frac{n+k}{2} L_{n-k}^{c} .}
\end{aligned}
$$


Moreover, the commutators of $L$ 's with $C$ 's read

$$
\begin{aligned}
{\left[C_{k}^{s}, L_{n}^{s}\right] } & =\frac{1}{8}\left((2 n-2 k-1) C_{k-2 n}^{s}+(2 n+2 k+1) C_{k+2 n}^{s}\right), \\
{\left[C_{k}^{s}, L_{n}^{c}\right] } & =\frac{1}{8}\left((-2 n+2 k+1) C_{k-2 n}^{c}+(2 n+2 k+1) C_{k+2 n}^{c}\right), \\
{\left[C_{k}^{c}, L_{n}^{s}\right] } & =\frac{1}{8}\left((2 n-2 k-1) C_{k-2 n}^{c}+(2 n+2 k+1) C_{k+2 n}^{c}\right), \\
{\left[C_{k}^{c}, L_{n}^{c}\right] } & =\frac{1}{8}\left((2 n-2 k-1) C_{k-2 n}^{s}-(2 n+2 k+1) C_{k+2 n}^{s}\right) .
\end{aligned}
$$

Summarizing, the symmetry algebra is defined by (4.14) and (4.15); as usual on the Lagrangian level there is no central change.

In order to have a better insight into this algebra let us note that the generators $L_{0}^{c}, L_{1}^{c}, L_{1}^{s}$ form a three-dimensional subalgebra:

$$
\left[L_{0}^{c}, L_{1}^{c}\right]=L_{1}^{s}, \quad\left[L_{1}^{s}, L_{0}^{c}\right]=L_{1}^{c}, \quad\left[L_{1}^{s}, L_{1}^{c}\right]=L_{0}^{c},
$$

in which, after the substitution,

$$
\tilde{H}=-\left(L_{1}^{c}+L_{0}^{c}\right), \quad K=L_{1}^{c}-L_{0}^{c}, \quad D=L_{1}^{s},
$$

we recognize the $\operatorname{sl}(2, R)$ algebra

$$
[D, \tilde{H}]=\tilde{H}, \quad[K, D]=K, \quad[\tilde{H}, K]=-2 D .
$$

Now, let us remind that the free classical motion (in general, in the sense of higher derivatives theory) possesses the conformal symmetry (i.e., $S L(2, \mathbb{R})$ symmetry, see [36]-[37]) which acts on time variable $\tilde{t}$ according to the formula

$$
\tilde{t}^{\prime}=\frac{a \tilde{t}+b}{c \tilde{t}+d}, \quad\left(\begin{array}{cc}
a & b \\
c & d
\end{array}\right) \in S L(2, \mathbb{R}) .
$$

This symmetry can be transformed to the harmonic oscillator case (the PU oscillator with the odd frequencies in the case of higher derivatives) by means of the Niederer transformation (or its generalization to the higher derivatives, see [23, 30] for further references) in which time of the free motion $\tilde{t}$ is related to "oscillator" time $t$ by the formula

$$
\tilde{t}=\tan (\omega t) .
$$


Since our model is a natural extension of the harmonic oscillator we can try to induce directly the symmetry from the action of $S L(2, \mathbb{R})$ on $\tilde{t}$. Indeed, taking the standard realization of the conformal generators

$$
\tilde{H}=-\sigma_{+}, \quad D=\frac{1}{2} \sigma_{3}, \quad K=\sigma_{-},
$$

we obtain, by virtue of eq. (4.19), the following infinitesimal action on $t$

$$
\begin{array}{ll}
\tilde{H}: & t^{\prime}=t-\frac{\epsilon}{2 \omega}(\cos (2 \omega t)+1), \\
K: & t^{\prime}=t+\frac{\epsilon}{2 \omega}(\cos (2 \omega t)-1), \\
D: & t^{\prime}=t+\frac{\epsilon}{2 \omega} \sin (2 \omega t),
\end{array}
$$

which perfectly agrees with the functions $f_{0}^{c}, f_{1}^{c}, f_{1}^{s}$ and the relation (4.17).

Moreover, the Hamiltonian (3.4) coincides with the generator of the time translation, i.e., $L_{0}^{c}=-\frac{1}{2 \omega} H$ which again agrees with the fact that the dynamics of the considered model is related to the different choice of the basis of the conformal algebra $H=\omega(\tilde{H}+K)$ (as in the case of the harmonic and PU oscillators, see [23, 48] and the references therein)

We see that $L$ 's define an infinite-dimensional extension of the $\operatorname{sl}(2, \mathbb{R})$ algebra. Things become more transparent if we use the functions $f_{k}(t)=$ $\frac{1}{2 \omega} e^{2 i k \omega t}$ with $k$-integer, instead of the functions (4.12), and $\psi_{k}$ (eq. (3.8)), with $k$ integer instead of the functions appearing in (4.4). Then the corresponding generators $L_{k}$ and $C_{k}$ (in the complexified algebra) can be expressed in the form

$$
L_{k}=L_{k}^{c}+i L_{k}^{s}, \quad C_{k}=\frac{1}{\sqrt{2 \alpha}}\left(C_{k}^{c}+i C_{k}^{s}\right), \quad k \text {-integer }
$$

and satisfy the following commutation relations

$$
\begin{aligned}
{\left[L_{k}, L_{n}\right] } & =i(k-n) L_{k+n}, \\
{\left[C_{k}, L_{n}\right] } & =\frac{i}{4}(2 k+1+2 n) C_{k+2 n} .
\end{aligned}
$$

Form eq. (4.25) we infer that the symmetry algebra can be split in two parts spanned by $C_{k}$ 's with $k$ even or odd, respectively; thus we define

$$
C_{k}^{+}=C_{2 k}, \quad C_{k}^{-}=C_{2 k-1}, \quad k \text {-integer. }
$$


As a result the commutation rules (4.25) are converted into the following ones

$$
\left[C_{k}^{ \pm}, L_{n}\right]=i\left(k+\frac{n}{2} \pm \frac{1}{4}\right) C_{k+n}^{ \pm}
$$

in which we recognize two representations of the Virasoro algebra acting on $C^{ \pm}$'s (with the nonhomogeneous part $\pm \frac{1}{4}$, see, e.g., [50]). At this point, let us recall that the PU oscillator with the odd frequencies enjoys the $\left(N-\frac{1}{2}\right)$ conformal non-relativistic symmetry which, for fixed $N$, possesses infinitedimensional extensions (see, [42]-[49]). However, there is no direct limit in these algebras as $N$ tends to infinity (in contrast to the Lagrangian or the Hamiltonian of the PU oscillator). They differ from the one considered above by the adjoint action of the Virasoro generators.

Finally, let us note that the model (3.2) as well as our former considerations can be extended to the case of coordinates taking their values in three-dimensional space $\vec{q}=\left(q^{\alpha}\right)$ (or, in generally, in $d$-dimensional one). Then the symmetry generators (4.6) and (4.13) extend naturally to the vector case: $C^{\alpha}$ 's and $L$ 's, respectively. Moreover, we have an additional family of generators

$$
J_{k}^{\alpha c}=f_{k}^{c} J^{\alpha}, \quad J_{k}^{\alpha s}=f_{k}^{s} J^{\alpha},
$$

related to the symmetry

$$
t^{\prime}=t, \quad \vec{q}^{\prime}=R(t) \vec{q},
$$

where $R$ is a time-depended rotation satisfying $R(t+\alpha)=R(t)$. As earlier (see (4.23) ) it is instructive to pass to the complexification of the algebra and define the new generators $J_{k}^{\alpha}=J_{k}^{\alpha c}+i J_{k}^{\alpha s}$. Then the commutation rules containing $J$ 's take the Kac-Moody form

$$
\begin{aligned}
{\left[J_{k}^{\alpha}, J_{n}^{\beta}\right] } & =\frac{1}{2 \omega} \epsilon^{\alpha \beta \gamma} J_{k+n}^{\gamma}, \\
{\left[J_{k}^{\alpha}, C_{n}^{\beta \pm}\right] } & =\frac{1}{2 \omega} \epsilon^{\alpha \beta \gamma} C_{n+k}^{\gamma \pm}, \\
{\left[J_{k}^{\alpha}, L_{n}\right] } & =i k J_{k+n}^{\alpha},
\end{aligned}
$$

which coincide with the ones described in refs. [42]-48 for a finite order conformal algebras (the difference is in the action of the Virasoro subalgebra). 


\section{Symmetry realizations on the Hamiltonian and quantum levels}

In this section we discuss the symmetry algebra in Hamiltonian approaches and show that it forms a natural central extension of the algebra on the Lagrangian level; on the quantum level the central extension appears also for the Virasoro algebra.

\subsection{Constrained approach}

First let us note that the Hamiltonian (3.4) as well as the symmetry generators $C$ 's and $L$ 's can be rewritten, due to the correspondence (2.6), as follows

$$
\begin{aligned}
H & =-\frac{m}{2 \alpha^{2}} \int_{-\alpha}^{\alpha} d \lambda Q(t, \lambda-\alpha) Q^{\prime}(t, \lambda), \\
L_{k}^{c} & =\frac{m \omega}{4 \pi^{2}} \int_{-\alpha}^{\alpha} d \lambda Q(t, \lambda-\alpha) Q^{\prime}(t, \lambda) \cos (2 \omega k(\lambda+t)), \\
L_{k}^{s} & =\frac{m \omega}{4 \pi^{2}} \int_{-\alpha}^{\alpha} d \lambda Q(t, \lambda-\alpha) Q^{\prime}(t, \lambda) \sin (2 \omega k(\lambda+t)), \\
C_{k}^{c} & =-\frac{m}{\alpha^{2}} \int_{-\alpha}^{\alpha} d \lambda Q(t, \lambda-\alpha) \cos \left(\frac{2 k+1}{2} \omega(\lambda+t)\right), \\
C_{k}^{s} & =-\frac{m}{\alpha^{2}} \int_{-\alpha}^{\alpha} d \lambda Q(t, \lambda-\alpha) \sin \left(\frac{2 k+1}{2} \omega(\lambda+t)\right) .
\end{aligned}
$$

Now, using the functional Dirac bracket (3.6) we can find all commutation rules and consequently the symmetry algebra on the Hamiltonian level. The computations are straightforward but rather tedious, due to the fact that we have to take carefully into account the support of each delta function appearing in the Dirac bracket (3.6). As a consequence, we obtain almost

the same relations on the Hamiltonian level as on the Lagrangian one - the only difference appears in commutators between $C$ 's; in this case we obtain a central extension, namely

$$
\left[C_{k}^{c}, C_{n}^{s}\right]=\frac{m}{\alpha}(-1)^{n-1} \delta_{n k}, \quad k, n=1,2,3 \ldots
$$




\subsection{Unconstrained approach}

As we saw in Section 3 solving the constraint (3.5) on the Hamiltonian level allow us to identify our model with the PU oscillator of the infinite order (see (3.12)). Therefore, it is instructive to find the form of the symmetry generators in terms of classical counterparts the creation and annihilation operators $c$ 's (equivalently $a$ 's ). We will see that the symmetry generators (except the Hamiltonian) are not direct extensions of the ones for the classical PU oscillator [22]. In order to do this we can express, by virtue of. eq. (3.7), the symmetry generators in terms of $a$ 's. After some troublesome calculations we arrive at the following form of the generators

$$
\begin{aligned}
C_{k}^{c} & =\sqrt{\frac{\alpha}{2}}\left(C_{k}+\bar{C}_{k}\right), \quad C_{k}^{s}=i \sqrt{\frac{\alpha}{2}}\left(\bar{C}_{k}-C_{k}\right), \\
L_{k}^{c} & =\frac{1}{2}\left(L_{k}+\bar{L}_{k}\right), \quad L_{k}^{s}=\frac{i}{2}\left(\bar{L}_{k}-L_{k}\right),
\end{aligned}
$$

where

$$
C_{k}=\frac{m}{\alpha^{2}} e^{\frac{i \pi}{2 \alpha}(2 k+1)(t-\alpha)} \bar{a}_{k}
$$

and

$$
\begin{aligned}
L_{n} & =-\frac{m}{8 \alpha^{2}} e^{2 i n \omega t} \sum_{k=-\infty}^{\infty}(-1)^{k}(2 k+1) a_{-k-2 n-1} a_{k} \\
& =\frac{m}{4 \alpha^{2}}(-1)^{n+1} e^{2 i n \omega t} \sum_{k=0}^{\infty}(-1)^{k}(2 k+1) \bar{a}_{k+n} a_{k-n} .
\end{aligned}
$$

The following identity appears to be useful

$$
\bar{a}_{k}=a_{-(k+1)} .
$$

Now, using the bracket (3.10) we can confirm the commutation relations (4.14), (4.15) and (5.2) obtained within the Hamiltonian framework with constraints. The calculations are straightforward but rather long so we only mention that, using (3.10) and (5.6), one can check the relation (4.24) which, in turn, easily implies (4.14).

Of course, one can express, by virtue of (3.11), the generators (5.4) and (5.5) in terms of $c^{\prime} s$ (for the Hamiltonian see (3.12)). Consequently we obtain a non-standard realization of the Virasoro algebra (4.24) what will be 
reflected in the form of the central extension on the quantum level (see (5.11) or (5.14) ). Moreover, let us note that

$$
\bar{C}_{n}=C_{-(n+1)}, \quad \bar{L}_{n}=L_{-n} \quad L_{0}=\bar{L}_{0}=L_{0}^{c}=-\frac{1}{2 \omega} H .
$$

Finally, the generalization of our considerations on the Hamiltonian level to the three-dimensional (in general $d$-dimensional ) case $Q^{\alpha}$ is straightforward and the main difference is that we have additional generators corresponding to the rotational symmetry (cf. eqs. (4.28) and (4.29)

$$
\begin{aligned}
J_{n}^{\alpha} & =\frac{m \omega}{4 \pi^{2}} \epsilon^{\alpha \beta \gamma} \int_{-\alpha}^{\alpha} d \lambda Q^{\beta}(t, \lambda-\alpha) Q^{\gamma}(t, \lambda) e^{2 i \omega n(t+\lambda)} \\
& =\frac{-i m \omega}{4 \pi^{2}} \epsilon^{\alpha \beta \gamma} e^{2 i \omega t n} \sum_{k=-\infty}^{\infty}(-1)^{k} a_{-k-2 n-1}^{\gamma} a_{k}^{\beta},
\end{aligned}
$$

which satisfy the commutation rules in (4.30).

\subsection{Quantum level}

In this section we analyse symmetries of the nonlocal PU model on the quantum level with special emphasis put on the Virasoro algebra. Following the standard reasoning we replace the classical variables $c_{k}, \bar{c}_{k}$, satisfying (3.13), by the creation and annihilation operators $\hat{c}_{k}, \hat{c}_{k}^{+}$

$$
\left[\hat{c}_{k}, \hat{c}_{n}^{+}\right]=\delta_{k n},
$$

for $k, n=0,1,2 \ldots$ Next, using the quantum counterpart of the relation (3.11) we define the operators $\hat{a}_{k}, \hat{a}_{-(k+1)}=\hat{a}_{k}^{+}$. Then we have the following commutator rules

$$
\left[\hat{a}_{k}, \hat{a}_{n}\right]=\frac{-\alpha^{2}}{m}(-1)^{k} \delta_{k+n+1,0}
$$

Now, we can translate the symmetry generators to the quantum level. In the case of $C^{\prime}$ 's, J's and $L_{n}$ with $n \neq 0$ it can be done directly (there is no problem with ordering of the operators, see eqs. (5.4), (5.5) and (5.8)); however, the generator $L_{0}$ requires more careful analysis. To this end let us compute the following commutator $\left[\hat{L}_{n}, \hat{L}_{-n}\right]$ for $n \neq 0$. Using the second form in (5.5) and (5.10) we arrive, after some computations, at the following result

$$
\left[\hat{L}_{n}, \hat{L}_{-n}\right]=-2 n \hat{L}_{0}+\frac{1}{12}\left(n^{3}-\frac{1}{4} n\right),
$$


where

$$
\hat{L}_{0}=\frac{-m}{4 \alpha^{2}} \sum_{k=0}^{\infty}(-1)^{k}(2 k+1) \hat{a}_{k}^{+} \hat{a}_{k} .
$$

Now let us express $\hat{L}_{0}$ in terms of the creation and annihilation operators

$$
\hat{L}_{0}=\frac{1}{4} \sum_{\substack{k=0 \\ k \text {-odd }}}^{\infty}(2 k+1) \hat{c}_{k}^{+} \hat{c}_{k}-\frac{1}{4} \sum_{\substack{k=0 \\ k \text {-even }}}^{\infty}(2 k+1) \hat{c}_{k} \hat{c}_{k}^{+} .
$$

Performing the regularization procedure by mens of the Riemann zeta function $\hat{L}_{0}$ becomes a normally ordered operator (proportional to the quantum Hamiltonian) and the following commutation rules

$$
\left[\hat{L}_{n}, \hat{L}_{k}\right]=(k-n) \hat{L}_{n+k}+\frac{1}{12}\left(n^{3}+\frac{3}{4} n\right) \delta_{k+n, 0},
$$

are satisfied; in the case of $d$ dimension there is the additional factor $d$ in front of the central charge in (5.14). Finally, by adding a constant to the normally ordered $\hat{L}_{0}$ and rescaling $\hat{L}_{n} \rightarrow-\hat{L}_{n}$ one obtains the standard form of the centrally extended Virasoro algebra.

\section{Conclusions}

We have discussed the symmetry properties of a simple nonlocal theory which provides the extension, to the case of an infinite number of degrees of freedom, of the PU oscillator with frequencies proportional to consecutive odd integers. Since the PU oscillator of order $N$ with such frequencies enjoys $\left(N-\frac{1}{2}\right)$-conformal non-relativistic symmetry the resulting symmetry algebra can be viewed as an extension of the latter one to infinite order $N=\infty$. It can be described within the framework of the Noether theorem both in its Lagrangian and Hamiltonian forms: the original $s l(2, \mathbb{R})$ subalgebra of conformal non-relativistic algebras is extended to the Virasoro algebra; the additional charges (outside the Virasoro subalgebra) form two representations of the latter under its adjoint action. They commute with each other on the Lagrangian level while on the Hamiltonian one we are dealing with the central extension defined by eq. (5.2). Moreover, on the quantum level the Virasoro algebra is also centrally extended (eq. (5.11) or (5.14)).

Let us note that the model described here resembles to some extent the string model. In the Hamiltonian framework we are dealing with string 
obeying antiperiodic boundary conditions (cf. eq. (3.5)) with the dynamics restricted to left-movers only (eq. (2.4)). There are, however, some important differences. First, $Q$ 's (or $q$ 's) take their values in Euclidean space and there are no additional constraints on them. Moreover, the energy is the alternating sum of independent oscillators energies (eq. (3.12)) which makes the system unstable and alters the vacuum energy. The model, although basically noninteracting, exhibits interesting structure. It is worthy of further study in several directions. First, one can consider the supersymmetric extensions following the construction proposed for finite order [24, 29]. Second, the above mentioned problem of (un)stability of the system (consequently the form of $\hat{L}_{0}$ ) might be studied along the lines proposed in ref. [14] for the quartic PU oscillator. According to this reasoning the unstability of the model could be cured at the expense of introducing negative norm states. Also the relation of our symmetry algebra to those considered in refs. [42][49] as well as a non-relativistic version of AdS $\backslash$ CFT correspondence for the discussed model are interesting questions.

Acknowledgments. Special thanks are to Piotr Kosiński who suggested the problem studied here. The discussions with Joanna and Cezary Gonera as well as Paweł Maślanka are gratefully acknowledged. The research was supported by the grant of National Science Center number DEC2013/09/B/ST2/02205.

\section{References}

[1] E. Witten, Nucl. Phys. B 268 (1986) 253

[2] D. Eliezer, R. Woodard, Nucl. Phys. B 325 (1989) 389

[3] N. Seiberg, E. Witten, JHEP 9909 (1999) 032

[4] J. Gomis, K. Kamimura, J. Llosa, Phys. Rev. D 63 (2001) 045003

[5] L. Joukovskaya, Phys. Rev. D 76 (2007) 105007

[6] T. Biswas, J. Kapusta, A. Reddy, JHEP 12 (2012) 008

[7] G. Calcagni, J. Phys. A47 (2014) 355402

[8] A. Pais, G. Uhlenbeck, Phys. Rev. 79 (1950) 145 
[9] J. Llosa, J. Vives, J. Math. Phys. 35 (1994) 2856.

[10] R. Woodard, Phys. Rev. A 62 (2000) 052105

[11] J. Gomis, K Kamimura, T. Mateos, JHEP 0103 (2001) 010

[12] J. Gomis, K. Kamimura,T. Ramirez, Nucl. Phys. B 696 (2004) 045003

[13] K. Bolonek-Lasoń, P. Kosiński, Act. Phys. Pol. B 45 (2014) 2057

[14] K. Andrzejewski, J. Gonera, P. Maślanka, Prog. Theor. Phys. 125 (2011) 247

[15] K. Andrzejewski, J. Gonera, P. Machalski, K. Bolonek-Lasoń, Phys. Lett. B 706 (2012) 427

[16] B. Bagchi, A. Choudhury, P. Guha, Mod. Phys. Lett. A 28 (2013) 1375001

[17] J. Jiménez, E. Di Dio, R. Durrer, JHEP 1304 (2013) 030

[18] S. Pramanik, S. Ghosh, Mod. Phys. Lett. A 28 (2013) 1350038

[19] M. Pavšič, Phys. Rev. D 87 (2013) 107502

[20] A. Galajinsky, I. Masterov, Phys. Lett. B 723 (2013) 190

[21] D. Kaparulin, S. Lyakhovich, A Sharapov, Eur. Phys. J. C 74 (2014) 3072

[22] K. Andrzejewski, Nucl. Phys. B 889 (2014) 333

[23] K. Andrzejewski, Phys. Lett. B 738 (2014) 405

[24] I. Masterov, Mod. Phys. Lett. A 30 (2015) 1550107

[25] A. Galajinsky, I. Masterov, Nucl. Phys. B 896 (2015) 244

[26] G. Pulgar, J. Saavedra, G. Leon, Y. Leyva, J. Cosmol. Astropart. Phys., (2015) 046

[27] J. Berra-Montiel, A. Molgado, E. Rojas, Ann. Phys. 362 (2015) 298 
[28] H. Kuwabara, T. Yumibayashi, H. Harada, preprint, Time dependent Pais-Uhlenbeck oscillator and its decomposition, arXiv:1503.03657

[29] I. Masterov, Nucl. Phys. B 902 (2016) 95

[30] K. Andrzejewski, A. Galajinsky, J. Gonera, I. Masterov, Nucl. Phys. B 885 (2014) 150

[31] J. Negro, M. del Olmo, A. Rodriguez-Marco, J. Math. Phys. 38 (1997) 3810

[32] C. Duval, P. Horvathy, J. Phys. A 42 (2009) 465206

[33] J. Lukierski, P. Stichel, W. Zakrzewski, Phys. Lett. A 357 (2006) 1

[34] J. de Azcaraga, J. Lukierski, Phys. Lett. B 678 (2009) 411

[35] S. Fedoruk, E. Ivanov, J. Lukierski, Phys. Rev. D 83 (2011) 085013

[36] J. Gomis, K. Kamimura, Phys. Rev. D 85 (2012) 045023

[37] K. Andrzejewski, J. Gonera, Phys. Lett. B 721 (2013) 319

[38] A. Galajinsky, I. Masterov, Nucl. Phys. B 866 (2013) 212

[39] N. Aizawa, Y. Kimura, J. Segar, J. Phys. A 46 (2013) 405204

[40] N. Aizawa, Z. Kuznetsova, F. Toppan, J. Math. Phys. 56 (2015) 031701

[41] N. Aizawa, Z. Kuznetsova, F. Toppan, preprint, Invariant PDEs of Conformal Galilei Algebra as deformations: cryptohermiticity and contractions, arXiv:1506.08488

[42] M. Henkel, J. Unterberger, Nucl. Phys. B 660 (2003) 407

[43] A. Bagchi, R. Gopakumar, JHEP 07 (2009) 037

[44] M. Alishahiha, A. Davody, A. Vahedi, JHEP 08 (2009) 022

[45] D. Martelli, Y. Tachikawa, JHEP 05 (2010) 091

[46] A. Hosseiny, S. Rouhani, J. Math. Phys. 51 (2010) 052307

[47] A. Bagchi, R. Gopakumar, I. Mandal, A. Miwa, JHEP 08 (2010) 004 
[48] A. Galajinsky, I. Masterov, Phys. Lett. B 702 (2011) 265

[49] C. Roger, J. Unterberger, The Schrödinger-Virasoro Lie group and algebra: Mathematical structure and dynamical Schrodinger symmetries, Springer, 2012.

[50] I. Kaplansky, Comm. Math. Phys. 86 (1982) 49 